\title{
Platelet Serotonin and Plasma Tryptophan in Depressed Patients: Effect of Drug Treatment and Clinical Outcome
}

\author{
Félicien Karege, Ph.D., Jean Widmer, Ph.D., Philippe Bovier, M.D., \\ and Jean-Michel Gaillard, M.D.
}

Platelet serotonin and plasma tryptophan were studied in healthy subjects and in depressed patients before and during their antidepressant drug treatment. Before treatment, mean platelet serotonin level was normal in depressed patients compared with healthy subjects while a significant decrease in patients' plasma TRP was noted $(\mathrm{t}=6.0, \mathrm{p}<.001)$. The concentrations of platelet $5-\mathrm{HT}$ level did not correlate with either plasma TRP or with clinical variables, that is, AMDP depression and AMDP anxiety scores. Antidepressant drugs treatment decreased platelet 5-HT level (ANOVA F $=8.27, \mathrm{p}<.001$ ) whatever the clinical outcome of the patient, whereas the changes observed in plasma TRP were positively related to the mood state change. These results suggest that platelet serotonin could be a good pharmacological model but has no relevance concerning the mood state.

[Neuropsychopharmacology 10:207-214, 1994]
KEY WORDS: Platelet serotonin; Plasma tryptophan; Depression; Antidepressants

Following the discovery that blood platelets accumulate serotonin (about $99 \%$ of total blood serotonin [5-HT] levels) due to their powerful uptake and storage mechanisms similar to those of central neurons (Stahl 1977), a large number of studies have examined the serotonin function of the blood platelet in various pathological conditions such as autism (Cook 1990), alcoholism (Bailly et al. 1990), and affective disorders (Quintana 1992). Clinical results from these different studies have

From the Institutions Universitaires de Psychiatrie de Genève (IUPG), Geneva, Switzerland.

Address correspondence to: Félicien Karege, Ph.D., Institutions Universitaires de Psychiatrie de Genève (IUPG), Service de la Recherche Biologique et de Psychopharmacologie clinique, 2 chemin du Petit Bel-Air, 1225 Chene-Bourg, Geneva, Switzerland.

Received August 16, 1993; revised January 10, 1994; accepted January 24, 1994. confirmed that blood platelet could be a convenient peripheral model for serotonin studies, and the advantage of its ease of preparation has contributed to popularize the use, with some limitations, of the platelet model (Pletscher and Laubscher 1980).

In affective disorders, attempts to determine the involvement of serotonin mechanisms have mainly focused on platelet 5-HT receptors (Arora and Meltzer 1989), ${ }^{3} \mathrm{H}$-imipramine binding (Langer and Briley 1981), and on the uptake mechanisms (Malmgren et al. 1981), where most of investigations have reported abnormalities. Very few studies have examined depression-associated changes in platelet 5-HT levels, and investigations in this domain have mostly yielded contradictory results (Lingjaerde 1979). For example, Mück-Seler et al. (1991) have recently reported a hyperserotonemia in unipolar depressed patients, whereas Quintana (1992) found a decreased platelet 5-HT content in major depressed patients. The same author also reported an increase of platelet 5-HT concentrations following imipramine treatment, whereas previous papers had often reported that imipramine or other tricyclics (Martensson et al. 1991; Celada et al. 1992) induced platelet 
5-HT depletion, whatever the clinical outcome. However, although early results from Wirz-Justice and Pühringer (1978a) reported an increase of platelet 5-HT in bipolar patients with no change in unipolar patients' platelet 5-HT levels, most later investigators (Sarrias et al. 1987) reported a decreased platelet 5-HT in melancholic patients, supporting the hypothesis that pathophysiology of some subtypes of depression is associated with serotonergic dysfunction (Coppen and Doogan 1988; Meltzer 1989) that could be also detected at the periphery. Nevertheless, blood 5-HT levels vary according to multiple physiological and pathological factors (Guicheney et al. 1988) and environmental factors such as photoperiod, season, or temperature (Sarrias et al. 1989). Moreover, although the central 5-HT originates exclusively from tryptophan directly decarboxylated by the enzymatic cell mechanisms, the platelet 5-HT origin is heterogenous, the enterochromaffin cells and the liver being the most important sources, rendering the platelet 5-HT levels sometimes inconsistent (Osim and Wyllie 1991).

With respect to plasma tryptophan (TRP) and affective disorders, a large number of studies were reported during the last two decades (Wood et al. 1978; Cowen et al. 1989), as the brain 5-HT was shown to depend on the plasma aminoacid precursors which cross the blood-brain barrier (Fernstrom 1983). Tryptophan circulates in blood plasma in two main forms, one free and one bound to plasma albumin. Changes in the plasma levels, free and total forms, have been described (Moller et al. 1979; Guicheney et al. 1988). The plasma TRP has been associated with mood changes (Young et al. 1988), and decreased plasma total TRP levels were previously reported in this laboratory (Bovier et al. 1988). It was thus suggested by some that, as a consequence of changes in plasma TRP levels, an altered amount of 5-HT occurs in brain and platelets (Quintana 1992). In spite of this suggested functional link of plasma TRP levels and functional serotonergic mechanisms in depression, no study has up to now successfully established a correlation between plasma TRP and platelet 5-HT levels.

The aim of this study was to provide additional data on platelet 5-HT and plasma TRP and to reassess the platelet 5-HT levels status in depressive syndromes and its relationships with plasma TRP, a subject that has received little attention. As longitudinal studies are required in studying relationships between biological variables and psychopathology, in a follow-up study we have investigated the correlations between serotonergic parameters, ATD drugs, and the change of mood. Lastly, comparisons between the effects induced by different classes of drugs were performed to complete our information in this subject.

\section{MATERIALS AND METHODS}

\section{Subjects}

Thirty depressed patients (20 F; $10 \mathrm{M}$ ) with mean age of $47 \pm 10$ years participated, with their consent, in this study. They met the criteria of DMS-III-R for depressive syndromes (24 major depression patients) recurrent or unique episode (293.6X); 3 bipolar depressed (296.5X) and 3 dysthymic patients (300.40) and were without any physical illness. At their hospitalization, patients frst underwent a drug washout period of a minimum of two weeks (14 to 26 days) before biochemical analysis.

The severity of depression was evaluated by two experienced clinicians using the AMDP depression rating scale (Bobon 1981) before treatment (T0) and after one month (T1), two months (T2), and three months (T3) of treatment. At the same time, the clinicians rated the severity of anxiety with the AMDP anxiety scale (Bobon 1981). At each time (T0, T1, T2, T3) patient's blood was collected and biochemical analysis, consisting of platelet serotonin and plasma tryptophan, was carried out. Only 28 patients receiving drug treatment were followed-up during the entire period of three months.

The treatment consisted of the various antidepressant drugs (ATD), randomly given in a daily dose of $150 \mathrm{mg}$ of imipramine to 12 patients; $300 \mathrm{mg}$ of fluvoxamine to two patients; 20 to $40 \mathrm{mg}$ fluoxetine to three patients; $600 \mathrm{mg}$ of moclobemid, a reversible monoamine oxidase inhibitor, to three patients; $250 \mathrm{mg}$ of maprotiline to three patients; and $150 \mathrm{mg}$ of amitriptyline to five patients.

Twenty subjects (10 F; $10 \mathrm{M}$ ) who served as controls in this study were in good physical health; they had no history of mental disorder, neurological disease, or drug abuse. Their mean age was $47 \pm 9$ years, the same as that of the patients.

\section{Blood Sampling}

Blood ( $9 \mathrm{ml}$ ) was drawn by venipuncture from the antecubital vein in $10-\mathrm{ml}$ tubes containing $1 \mathrm{ml}$ of anticoagulant (ACD, $0.06 \mathrm{M}$ citrate- $\mathrm{Na} ; 0.038 \mathrm{M}$ citric acid and $0.136 \mathrm{M}$ dextrose). Blood collection was always performed in morning between 8 and 9 A.M., and the platelet rich plasma (PRP) was isolated within 30 minutes by centrifugation $(160 \mathrm{~g} \times 10$ minutes $)$ at $4^{\circ} \mathrm{C}$. A small aliquot $(100 \mathrm{ml})$ was taken to count the platelet number.

For the study of platelet 5-HT, the PRP was centrifuged again at $5100 \mathrm{~g} \times 10$ minutes to pellet platelets. The platelets were resuspended in a washing buffer $(50 \mathrm{mM}$ Tris- $\mathrm{HCl})$ and washed before another spin at the above speed. Then the pelleted platelets were lysed in $0.1 \mathrm{M} \mathrm{H}_{3} \mathrm{PO}_{4}$ by sonication and centrifuged again. 
Table 1. Platelet 5-HT Concentrations (Mean \pm SEM) and Plasma Total Tryptophan $( \pm$ SEM) in 20 Control Subjects and in 30 Drug-free Depressed Patients

\begin{tabular}{lccc}
\hline & $\begin{array}{c}\text { Platelet 5-HT } \\
\left(\mathbf{n g} / \mathbf{1 0}^{\mathbf{9}} \text { platelet) }\right.\end{array}$ & $\begin{array}{c}\text { Plasma TRP } \\
(\boldsymbol{\mu g} / \mathbf{m l} \text { plasma) }\end{array}$ & $\begin{array}{c}\text { Platelet Count } \\
\mathbf{1 0}^{\mathbf{9}} \text { plat/1 }\end{array}$ \\
\hline Controls (20) & $1.307 \pm 0.14$ & $11.84 \pm 0.3$ & $429 \pm 15$ \\
Patients (30) & $1.405 \pm 0.18$ & $8.52 \pm 0.4^{\star \star \star}$ & $369 \pm 19^{\star}$ \\
\hline
\end{tabular}

Platelet 5-HT level and plasma TRP were measured by HPLC on platelets isolated from blood sample collected at 8 A.M. Statistical analysis was performed with Student $t$ test to compare controls, and patients gave significant differences in plasma TRP and in platelet count.

${ }^{\star} t=-2.31, p<.03$.

$\star \star \star t=6.00, p<.001$.

The supernatant was isolated and injected in a HPLC apparatus with an electrochemical detector set at 0.72 $\mathrm{mv}$ of potential. Results were expressed in $\mathrm{ng} / 10^{9}$ platelets.

For the plasma tryptophan study, the platelet poor plasma (PPP) was isolated either from PRP or from whole blood by centrifugation at $5100 \mathrm{~g} \times 10$ minutes at $4^{\circ} \mathrm{C}$. $0.1 \mathrm{ml}$ plasma was deproteinezed with 1.1 $\mathrm{mmol} / \mathrm{L}$ perchloric acid $(0.85 \mathrm{ml})$, neutralized with $15 \mathrm{~N}$ $\mathrm{KOH}(0.05 \mathrm{ml})$ then centrifuged, and the supernatant was injected in a HPLC apparatus with a UV-Visible detector set at $206 \mathrm{~nm}$. Tryptophan was coeluted with other neutral aminoacid, phenylethylamine, and tyrosine on the same chromatograph. Results were expressed in $\mathrm{mg} / \mathrm{ml}$ plasma. The statistical analysis was performed using a two-way ANOVA between groups, followed by Student $t$ test, and in some cases regression analysis was performed for the calculation of correlations between parameters. Statistical significance was set at $p<.05$.

\section{RESULTS}

Table 1 shows the mean values of platelet 5-HT levels and plasma TRP levels in drug-free depressed patients. With respect to platelet 5-HT level there was no signifi- cant difference between patients and control subjects ( $t$ test $t=.67, \mathrm{NS}$ ). For plasma TRP, patients presented significantly decreased values compared to controls ( $t$ test $t=6.0, p<.001)$ and a low platelet count $(t$ test $t=2.13, p<.03$ ) (Table 1). The exclusion of non unipolar patients did not change the results because no difference was observed in the diagnostic groups.

Although a tendency for male subjects to present relatively higher TRP levels than females was previously reported, the difference between both sexes was not significant in this population. In normal controls the TRP mean value $(X \pm S E M)$ was $12.15 \pm 0.5 \mu \mathrm{g} / \mathrm{ml}$ and $11.68 \pm 0.5 \mu \mathrm{g} / \mathrm{ml}$ for males and females, respectively ( $t$ test was $1.01, \mathrm{NS}$ ) and in patients, the mean value was $8.67 \pm 0.4 \mu \mathrm{g} / \mathrm{ml}$ and $8.32 \pm 0.4 \mu \mathrm{g} / \mathrm{ml}(t$ test was 0.93 , NS) for males and females, respectively.

Furthermore, the analysis of other neutral aminoacids revealed no significant difference between patients and controls. Plasma tyrosine mean $(X \pm S E M)$ level was $11.26 \pm 0.3 \mu \mathrm{g} / \mathrm{ml}$ and $10.92 \pm 0.3 \mu \mathrm{g} / \mathrm{ml}$ ( $t$ test: NS) for controls and patients respectively. Plasma phenylalanine mean $(X \pm S E M)$ level was $9.16 \pm$ $0.4 \mu \mathrm{g} / \mathrm{ml}$ in controls versus $9.52 \pm 0.5 \mu \mathrm{g} / \mathrm{ml}$ in depressed patients ( $t$ test: NS).

Correlations between biochemical and clinical variables were calculated, and the results are summarized on Table 2. Platelet 5-HT levels did not correlate with either plasma TRP or clinical parameters $(r=.18, \mathrm{NS}$,

Table 2. Correlation Coefficients $(r)$ between Platelet 5-HT Levels, Plasma Tryptophan, Clinical and Demographic Variables

\begin{tabular}{|c|c|c|c|c|}
\hline & $\begin{array}{l}\text { Plasma } \\
\text { TRP }\end{array}$ & $\begin{array}{c}\text { AMDP } \\
\text { Depression }\end{array}$ & $\begin{array}{c}\text { AMDP } \\
\text { Anxiety }\end{array}$ & Age \\
\hline $\begin{array}{l}\text { Platelet 5-HT } \\
\text { Plasma TRP }\end{array}$ & $r=0.05 ; \mathrm{NS}$ & $\begin{array}{l}r=0.18 ; \mathrm{NS} \\
r=0.02 ; \mathrm{NS}\end{array}$ & $\begin{array}{l}r=0.03 ; \mathrm{NS} \\
r=0.05 ; \mathrm{NS}\end{array}$ & $\begin{array}{l}r=0.19 ; \text { NS } \\
r=0.31, p<0.07\end{array}$ \\
\hline
\end{tabular}

The correlation coefficient was calculated by the regression analysis method to establish possible relationships between biochemical and clinical variables. No statistically significant correlation coefficient was obtained. Moreover, male and female patients' platelet 5-HT means levels were not statistically different $(t$ test $=$ NS) 
Table 3. Mean Values ( \pm SEM) of Biological Variables (Platelet 5-HT, Platelet Number and Plasma TRP) and Clinical Variables (AMDP Depression and AMDP Anxiety) before Treatment (T0) and Their Changes after One Month (T1), Two Months (T2), and Three Months (T3) of Treatment, Respectively.

\begin{tabular}{lcccr}
\hline & T0 & T1 & T2 & T3 \\
\hline Platelet 5-HT (a) (ng/10 ${ }^{9}$ plat.) & $1.405 \pm 0.18$ & $0.722 \pm 0.19^{\star \star \star}$ & $0.452 \pm 0.15^{\star \star \star}$ & $0.255 \pm 0.06^{\star \star \star}$ \\
Platelets count (b) & $369 \pm 19$ & $408 \pm 20$ & $382 \pm 21$ & $353 \pm 17$ \\
$\quad\left(10^{9}\right.$ plat./I) (30) & $8.57 \pm 0.3$ & $9.93 \pm 0.4^{\star}$ & $10.14 \pm 0.5^{\star}$ & $10.31 \pm 5^{\star}$ \\
Plasma TRP (c) $(\mu \mathrm{g} / \mathrm{ml})$ & $22.3 \pm 1.8$ & $12.9 \pm 2^{\star \star}$ & $11.7 \pm 2^{\star \star \star}$ & $9.16 \pm 2.0^{\star \star \star}$ \\
AMDP depression (d) & $5.36 \pm 0.5$ & $3.42 \pm 0.5^{\star}$ & $3.88 \pm 0.6$ & $2.14 \pm 0.4^{\star \star}$ \\
AMDP anxiety (e) & &
\end{tabular}

Thirty depressed patients were examined at T0, but only twenty-eight of them were examined during the treatment period.

Statistical comparisons were performed with a two-way ANOVA for each parameter between different times (T0 to T3) of study, followed by the Student $t$ test between the different treatment values, with respect to the pre-treatment value (T0): ${ }^{\star} p<.05,{ }^{\star \star} p<.001$, ${ }^{\star \star \star} p<.001$. ANOVA: (a) $\mathrm{F}=8.27, p<.001$; (b) $\mathrm{F}=0.6$, NS; (c) $\mathrm{F}=2.14, p<.05$; (d) $\mathrm{F}=6.29, p<.001 ;(\mathrm{e}) \mathrm{F}=4.51, p<.001$.

and $r=.03$, NS for depression and anxiety, respectively), and no effects of age $(r=.19$, NS) or sex were evident. Similarly, plasma TRP did not correlate to the AMDP depression $(r=.02$, NS), anxiety $(r=.05$, NS), age $(r=.31, p<.07)$, and sex (Table 2$)$.

Changes that occurred during the period of ATD drug treatment in platelet 5-HT levels and in plasma TRP were measured (Table 3). From T0 to T3, platelet 5-HT levels underwent a drastic decrease (ANOVA: $\mathrm{F}=$ $8.27, p<.001)$ whereas plasma TRP slightly increased during the same period (ANOVA: $\mathrm{F}=2.14, p<.05$ ). The clinical parameters (AMDP depression and AMDP anxiety mean scores) also significantly decreased in the same period (ANOVA F $=6.29, p<.001$ and $F=4.51$, $p<.001$, respectively). In the first month of treatment (T1), about $50 \%$ of platelet 5 -HT level was depleted ( $t$ test $t=3.09, p<.0001$ ), and AMDP depression score was approximately decreased by $50 \%$ ( $t$ test $t=3.36$, $p<.001$ ). After three months, the platelet 5-HT levels represented only about $15 \%$ of the pretreatment value ( $t$ test $t=4.07, p<.001$ ), and AMDP depression and anxiety had progressively decreased. The plasma TRP level increased modestly and did not reach the control values. No change was observed in platelet number during the follow-up period (ANOVA $F=0.6, N S$ ).

The clinical outcome and the effect of drugs on biochemical parameters were examined to understand the changes observed in platelet 5-HT level and in plasma TRP. With respect to clinicaloutcome, Figure 1A shows that no difference was observed in the evolution of platelet 5-HT. In both improved (more than $50 \%$ change in AMDP depression score) and nonimproved (less than $50 \%$ change in AMDP depression score), the decrease in platelet 5-HT was the same, whereas plasma TRP (Figure 1B) increased only in improved patients (ANOVA: $\mathrm{F}=3.62 ; p<.002$ and $\mathrm{F}=1.5 ; \mathrm{NS}$ ). The difference in AMDP anxiety score between improved and nonimproved patients was also significant, and the change was only observed in improved patients $(\mathrm{F}=6.7 ; p<.001$ and $\mathrm{F}=1.5 ; \mathrm{NS}$ for improved and nonimproved, respectively). Improved patients were less anxious than nonimproved subjects ( $t$ test: $p<.01)$ (Fig. 1C).

To investigate the effects of drugs on platelet 5- $\mathrm{HT}$ changes, the different antidepressants used in the treatment were grouped in four categories: selective 5-HT blockers (fluoxetine and fluvoxamine); b-selective NA blockers (maprotiline); mixed 5-HT/NA blockers (imipramine, amitriptyline); and monoamineoxidase inhibitors (moclobemid). Figure 2 shows that important change occurred in platelet 5-HT of patients treated with serotonergic or "mixed" drugs whereas no change was observed in NA blockers drugs (ANOVA F $=14.06$; $p<.001$ and $\mathrm{F}=12.06 ; p<.001$ for the "mixed" and the serotonergic drug group, respectively). In contrast, the patients who were treated with moclobemid (a MAOI) or mapropotiline (a noradrenergic drug) did not significantly change their mean values during the treatment period (ANOVA $\mathrm{F}=0.17, \mathrm{NS}$ and $\mathrm{F}=0.3$, NS for MAOI- and moclobemid-treated patients, respectively). Yet a small increase of platelet 5-HT level was observed at $\mathrm{T} 1$ in the MAOI-treated group (the nonparametric Wilcoxon test yielded $\mathrm{Z}=-2.02 ; p<.04)$.

\section{DISCUSSION}

The present study indicated first that platelet 5-HT content was not different in drug-free depressed patients, compared to normal controls, whereas plasma TRP levels were significantly decreased in patients. Furthermore, no difference was observed in male and female subjects, both in controls and patients group of this population with respect to their plasma TRP level. The other plasma neutral aminoacids, that is, phenylalanine and tyrosine, co-analyzed with TRP did not present any difference between normal controls and patients.

Second, both serotonergic indices (i.e., platelet 5-HT levels and plasma TRP) did not correlate either with each other, in spite of their precursor-metabolite 

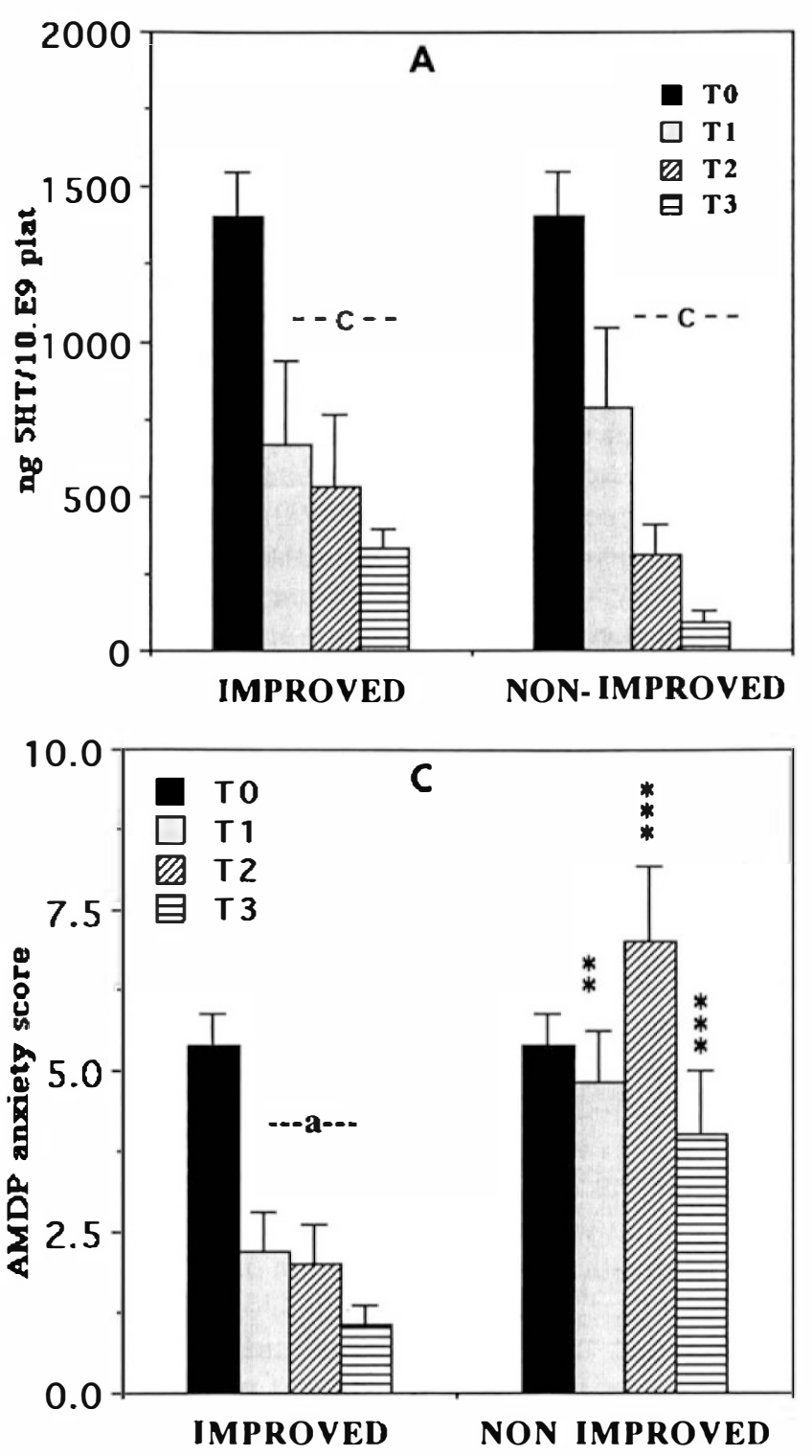

affiliation, or to clinical rating scores (i.e., AMDP depression and anxiety).

Third, serotonergic and "mixed" NA/5-HT ATD drugs treatment induced a marked decrease in platelet 5-HT stores, independently of the clinical outcome, whereas plasma TRP changes were associated to the mood state changes. Moreover, it should be mentioned that no difference in platelet 5-HT or plasma TRP was found between male and female subjects, and no effect of age was detected in accordance to previous reports (Stahl et al. 1983).

The lack of change in platelet 5-HT level of depressed patients observed in this study was at variance with some previous studies (Le Quan-Bui et al. 1984, Sarrias et al. 1987, or Quintana 1992) that reported a decrease in platelet 5 -HT content in major depression. The reason for this discrepancy is unclear. Several factors are involved in the control of the platelets store,

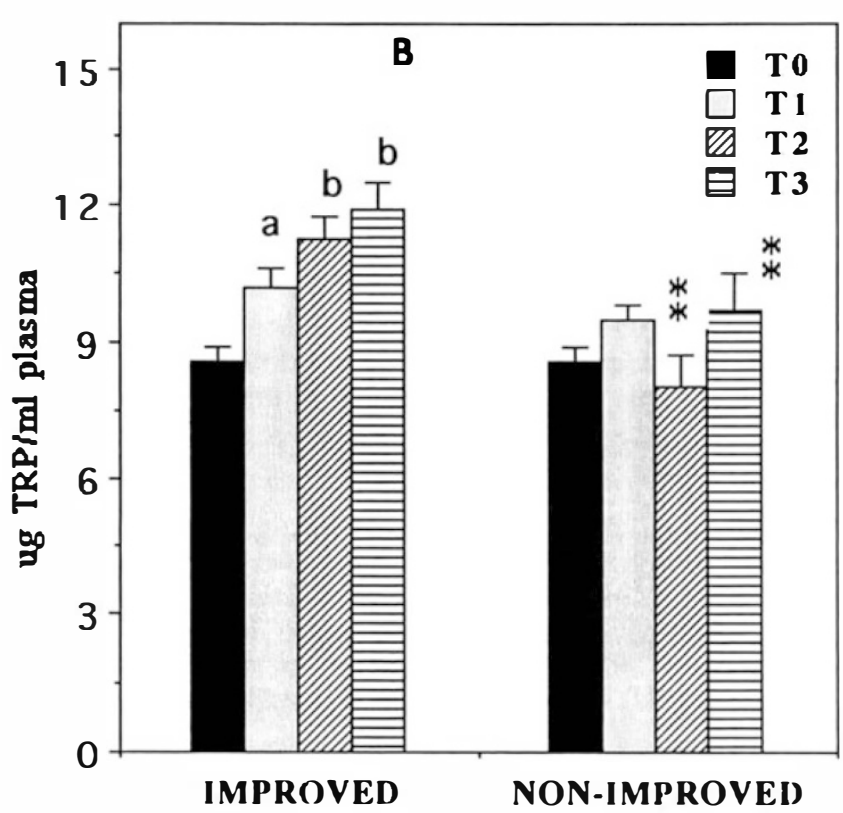

Figures 1. Changes in platelet 5-HT levels (A), plasma tryptophan (B) and AMDP anxiety scores (C) with respect to clinical improvement. Improvement in depression was arbitrarily set at $>50 \%$ of AMDP depression score decrease. Statistical analysis was performed with a two-way ANOVA followed by a Student $t$ test to compare improved and non-improved patients or drug treated to drug-free patients. The test of ANOVA for both groups with regard to 5-HT: $F=5.68, p<$ .001 and $F=4.89 p<.001$ for improved and nonimproved patients respectively $(\mathrm{A})$, with regard to plasma TRP: $F=3.62$; $p<.02$ and $F=1.51$; NS for improved and nonimproved respectively $(B)$ and with regard to AMDP anxiety change: $F=6.71 ; p<.001$ and $F=1.53$; NS for improved and non improved respectively (C). Student $t$ test between improved and nonimproved: ${ }^{\star \star} p<.01 ;{ }^{\star \star \star} p<.001$. Student $t$ test between drugs treated (T1 to T3) to drug-free (T0) patients: A, $p<.05 ; \mathrm{B}, p<.01 ; \mathrm{C}, p<.001$.

such as the uptake mechanism by platelet membrane, the storage processes in dense granules, the process of release, and the intracellular conjugation or degradation of the amine (Le Quan-Bui et al. 1983). Any alteration in the above factors should affect platelet stores; indeed the uptake mechanism was observed by most (Tuomisto and Tukiainen 1976, Malmgren et al. 1981) but not all investigators (Stahl et al. 1983) to be decreased in platelets from unipolar depressed patients. However, there are also reports at variance with the depression-associated reduction of platelet 5-HT level. Wirz-Justice and Pühringer (1978a) have found an increase in bipolar patients without alteration in absolute platelet 5-HT levels from unipolar patients, and those results were later replicated by Stahl et al. (1983). Moreover, the study from Mück-Seler et al. (1991) was also at variance with the reduced platelet 5-HT level in endogenous depression (Mück-Seler et al. 1991). These 


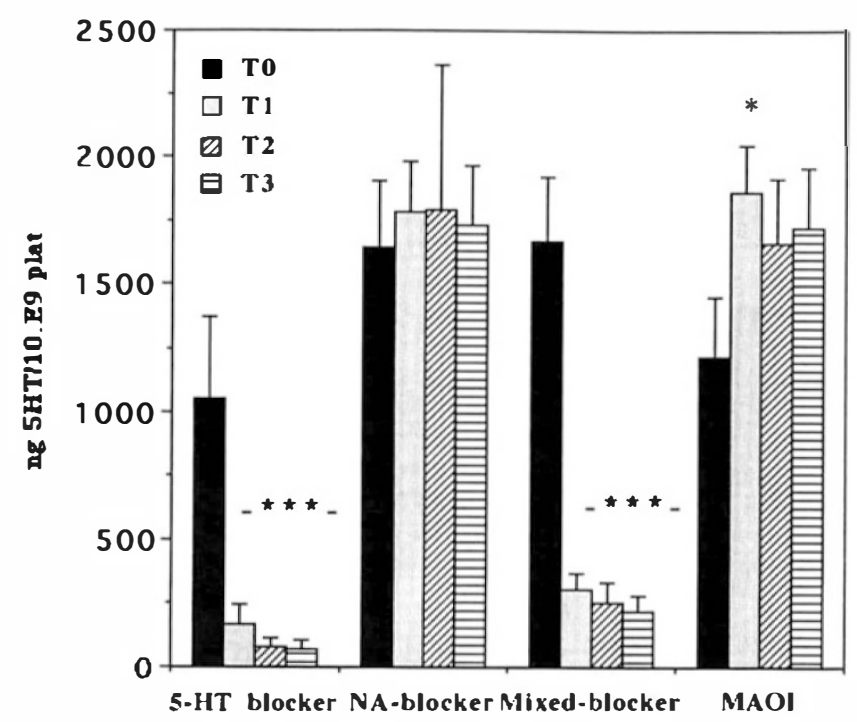

Figure 2. Changes in platelet 5-HT levels with respect to the type of antidepressant drug administered during the treatment period. ATD drugs were grouped in four categories according to their mode of action, namely: 5 -HT blockers, fluoxetine or fluvoxamine ( 5 patients); NA blocker, maprotiline ( 3 patients); 5-HT/NA mixed blockers, imipramine and amitriptyline (17 patients); MAOI, meclobemid (3 patients). The daily dosages for each drug are given in "Material and Methods." Statistical analyses performed with either a two way ANOVA $\left({ }^{\star \star \star}\right)$ : 5-HT antidepressants group: $F=12.04 ; p<.01$; Mixed antidepressants group $F=14.06 ; p<.001$; or a Wilcoxon nonparametric test between $\mathrm{T} 1$ and $\mathrm{T} 0\left({ }^{\star}\right)$ : MAOI antidepressant, $Z=2.02, p<.04$.

authors reported a hyperserotonemia in major depression. The results indicate that the status of the platelet 5 -HT level is not yet clear. Differences resulting in nonhomogeneity of patient groups and assay problems related to platelets preparation cannot alone account for those inconsistent findings. According to Ortiz et al., the blood contains two different pools of serotonin, the plasma 5-HT, in nanomolar concentrations, which is a rapid turn-over pool more affected by short-term variations in 5-HT physiology, and the platelet 5-HT in micromolar concentrations, which constitutes a reserve with a slow turnover pool and sensitive to more sustained changes (Ortiz et al. 1988). Future results will indicate which of those blood serotonergic pools would be more functionally linked to psychopathological states.

With regard to plasma TRP, our study has found lowered level in depression with a tendency to normalize in drug treatment responders. This reduced plasma TRP in depression was previously reported by others (Coppen et al. 1973; Cowen et al. 1989). In drug responders, the observed increase of plasma TRP could be attributed to the recovery of the mechanisms of aminoacid synthesis. But paradoxically, this newly syn- thesized TRP was not reflected in the platelet 5-HT content. This is due to the presence of antidepressant drugs. However, other neutral aminoacids (NAA) have been shown to compete with TRP in transport membrane, and the ratio of TRP to the NAA, especially tyrosine and phenylalanine, could influence the synthesis of 5-HT (Wurtman and Fernstrom 1972; Acwort et al. 1988). Plasma TYR and PHE have been measured in the present study, and no change was found between endogenous depressed patients and normal controls. Moreover, as we showed previously, plasma TYR and PHE levels did not change during a six-week period of drug treatment (Chiaroni et al. 1990). Thus, the probability that these aminoacids influenced the synthesis of 5 -HT is very low, but caution must be taken, as all of the NAAs (i.e., leucine, isoleucine and valine) have not been measured.

In addition, the plasma TRP level did not increase the normal values, and in this sense it would resemble other serotonergic parameters like ${ }^{3} \mathrm{H}$-imipramine binding (Langer and Briley 1981), and CSF 5-HIAA (Traskman-Bendz et al. 1984). Thus, the lack of correlation between platelet 5-HT and plasma TRP and the lack of effect of plasma TRP changes on platelet 5-HT render questionable the relevance of platelet 5-HT in depression. Studying the source of serotonin in blood, Pietraszek et al. have recently reported that brain 5-HT was correlated to both blood and brain TRP pools, whereas 5-HT was not (Pietraszek et al. 1992). The blood serotonin was only correlated to that in stomach; this could partially explain the above results.

The drastic decrease observed in platelet 5-HT level under "mixed" (imipramine and chlomipramine) and serotonergic drugs (fluvoxamine and fluoxetine) is in agreement with previous reports (Celada et al. 1991; Sarrias et al. 1989) and should be interpreted as a consequence of the action of these drugs in inhibiting the uptake of 5-HT levels. The entry of 5-HT into the cell is still blocked by the presence of ATD and conversely, newly formed platelets contain very low levels of the amine. Moreover, this decrease was of the same extent in both drug-responders and nonresponder patients. As in the above result, this point reinforces the doubt about the relevance of the platelet 5-HT levels in depression. It could point out, a pharmacological action of the antidepressants, however, specifically drug-associated as no decrease was observed in the noradrenergic-drug (maprotiline)-treated patients. Thus, platelets would be a good index of the pharmacological efficacy in regard to 5-HT uptake. The inhibition of amine oxidation (with moclobemid), as expected, induced a slight increase at T1 in the platelet 5-HT content. This indicates that, in the absence of blockers with a serotonergic action, mechanisms of 5-HT entering the cell were still working, and the inhibition of oxidation contributed to the relative increase in the amine level. The results are com- 
parable with brain studies where it was reported that long term re-uptake inhibition, but not monoamine oxidase inhibition, actually decreases the 5-HT function (Blier et al. 1988).

In conclusion, this study has noted a decreased plasma TRP with no change in platelet 5-HT level in depressed patients. Although both measures were not correlated to depression severity or to anxiety scores, plasma TRP was more associated with depressive state than was platelet 5-HT because it changed in relation to depression severity and it trended to normalization in ameliorated patients only. ATD drugs induced a change in platelet 5-HT level specifically related to the mode of drug action, and this could be of pharmacological significance.

\section{REFERENCES}

Acwort IN, During MJ, Wurtman RJ (1988): Process that couple aminoacid availability to neurotransmitter synthesis and release. In G. Huether (ed), Aminoacid Availability and Brain Function in Health and Disease. Berlin: Springer-Verlag, pp 117-136

Arora RC, Meltzer HY (1989): Increased serotonin-2 (5-HT $)$ receptor-binding as measured by $\left({ }^{3} \mathrm{H}\right)$-lysergic acid $\mathrm{di}$ ethylamide ( ${ }^{3} \mathrm{H}$-LSD) in the blood-platelets of depressed patients. Life Sci 44:725-734

Bailly D, Vignau J, Lauth B, Racadot N, Beuscart R, Servant D, Parquet PJ (1990): Platelet serotonin decrease in alcoholic patients. Acta Psychiatr Scand 81:68-72

Blier P, Chaput Y, de Montigny C (1988): Long-term reuptake blockade, but not monoamine oxidase inhibition, decreases the function of terminal 5-HT autoreceptors: an electrophysiological study in the rat brain. NaunynSchimiedeberg's Arch Pharmacol 337:246-254

Bobon DP (1981): Le Système AMDP. Manuel de Documentation et de Quantification de la Psychopathologie, 2nd ed, Brussels: Mardaga $P$

Bovier P, Widmer J, Gaillard JM, Tissot R (1988): Evolution of red blood cell membrane transport and plasma level of L-tyrosine and L-tryptophan in depressed treated patients according to clinical improvement. Neuropsychobiology 19:125-134

Celada P, Dolera M, Alvarez E, Artigas F (1992): Effects of acute and chronic treatment with fluvoxamine on extracellular and platelet serotonin in the blood of major depressive patients. Relationship to clinical improvement. J Affective Disorders 25:243-250

Chiaroni P, Azorin JM, Bovier P, Widmer J, Jeanningros R, Barré A, Dufour H, Tatossian A, Tissot R (1990): A multivariate analysis of red blood cell membrane transports and plasma levels of L-tyrosine and L-tryptophan in depressed patients before treatment and after clinical improvement. Neuropsychobiology 23:1-7

Cook EH (1990): Autism: review of neurochemical investigation. Synapse 6:292-308

Coppen A, Eccleston EG, Peet M (1973): Total and free tryp- tophan concentration in the plasma of depressive patients. Lancet II:60-63

Coppen SE, Kirk L, Honoré P (1979): Free and total plasma tryptophan in endogenous depression. J Affective Disorders 1:69-76

Coppen AJ, Doogan DP (1988): Serotonin and its place in the pathogenesis of depression. J Clin Psychiatry S49:4-11

Cowen PJ, Parry-Billings M, Newsholme EA(1989): Decreased plasma tryptophan levels in major depression. J Affective Disorders 16:27-31

Fernstrom JD (1983): Role of precursor availability in control of monoamine synthesis in brain. Physiol Rev 63:484-546

Guicheney P, Leger D, Barrat J, Treveux R, De Lignieres B, Roques P, Garmier JP, Boyer P, Grenier J, Dreux C. (1988): Platelet serotonin content and plasma tryptophan in periand postmenopausal women: variations with plasma oestrogen levels and depressive symptoms. Eur J Clin Invest 18:297-304

Langer SZ, Briley M (1981): High-affinity ${ }^{3} \mathrm{H}$-IMI binding: new biological tool for studies in depression. Trends Neurosci 4:28-31

Le Quan-Bui KH, Plaisant O, Leboyer M, Gay C, Kamal L, Devynck MA, Meyer P (1984): Reduced platelet serotonin in depression. Psychiatry Res 13:129-139

Lingjaerde O (1979): Le rôle de la sérotonine dans les troubles de l'humeur. L'Encéphale 5:499-506

Malmgren R, Asberg M, Olsson P, Tornling G, Unge G (1981): Defective serotonin transport mechanism in platelets from endogenously depressed patients. Life Sci 29: 2649-2658

Martensson B, Wägner A, Beck O, Brodin K, Montero D, Asberg M (1991): Effects of chlomipramine treatment on cerebrospinal fluid monoamine metabolites and platelet ${ }^{3} \mathrm{H}$-imipramine binding and serotonin uptake and concentration in major depressive disorder. Acta Psychiatr Scand 83:125-133

Meltzer HY (1989): Serotonergic dysfunction in depression. Br J Psychiatry 8:25-31

Mück-Seler D, Jakovljevic M, Deanovic Z (1991): Effect of antidepressant treatment on platelet 5 -HT content and relation to therapeutic outcome in unipolar depressive patients. J Affective Disorders 23:157-164

Ortiz J, Artigas F, Gelpi E (1988): Serotonergic status in human blood. Life Sci 43:983-990

Osim EE, Wyllie JH (1991): Blood platelet serotonin following enterectomy in rats. Acta Physiologica Hungarica 78:173-179

Pietraszek MH, Takada Y, Yan D, Urano T, Serizawa K, Takada A (1992): Relationship between serotonergic measures in periphery and the brain of mouse. Life Sciences 51:75-82

Pletscher A, Laubscher A (1980): Blood platelets as models for neurons: uses and limitations. J Neural Transm $16: 7-16$

Quintana J (1992): Platelet serotonin and plasma tryptophan decreases in endogenous depression. Clinical, therapeutic, and biological correlations. J Affective Disorders 24: 55-62

Sarrias MJ, Artigas F, Martinez E, Gelpi E, Alvarez E, Udina C, Casas M (1987): Decreased plasma serotonin in melan- 
cholic patients: a study with clomipramine. Biol Psychiatry 22:1429-1438

Sarrias MJ, Artigas F, Martinez E, Gelpi E (1989): Seasonal changes of plasma serotonin and related parameters: correlation with environmental measures. Biol Psychiatry 26:695-706

Stahl SM (1977): The human platelet: a diagnostic and research tool for the study of biogenic amines in psychiatric and neurologic disorders. Arch Gen Psychiatry 34:509-516

Traskman-Bendz L, Asberg M, Bertilsson L, Thoren P (1984): CSF monoamine metabolites of depressed patients during illness and after recovery. Acta Psychiatr Scand 69:333-342

Tuomisto J, Tukiainen E (1976): Decreased uptake of 5-hy- droxytryptamine in blood platelets from depressed patients. Nature 262:596-598

Wirz-Justice A, Pühringer W (1978a): Increased Platelet Serotonin in Bipolar Depression and Hypomania. J Neural Transmission 42:55-62

Wirz-Justice A, Pühringer W (1978b): Seasonal incidence of an altered diurnal rhythm of platelet serotonin in unipolar depression. J Neural Transmission 42:45-53

Wurtman RJ, Fernstrom JD (1972): L-tryptophan, L-tyrosine and the control of brain monoamine biosynthesis. In Sinder SH (ed), Perspectives in Neuropharmacology. New York: Oxford University Press, pp 143-193

Young SN, Pihl RO, Ervon FR (1988): The effect of altered tryptophan levels on mood and behavior in normal human males. Clin Neuropharmacol Suppl 1:207-215 